\title{
ESPR uroradiology task force and ESUR paediatric working group: imaging and procedural recommendations in paediatric uroradiology, part III. Minutes of the ESPR uroradiology task force minisymposium on intravenous urography, uro-CT and MR-urography in childhood
}

\author{
Michael Riccabona • Fred E. Avni - Jean-Nicholas Dacher • Maria Beatrice Damasio • \\ Kassa Darge • M. Luisa Lobo • Lil-Sofie Ording-Müller • Frederika Papadopolou • \\ Ulrich Willi
}

Received: 15 February 2010 / Accepted: 16 March 2010/Published online: 22 May 2010

(C) Springer-Verlag 2010

\begin{abstract}
The ESPR working groups have addressed intravenous urography (IVU), uro-CT and MR-urography (MRU) in childhood as the last in the series of recommendations for paediatric uroradiology. The aim of this process was again to standardise paediatric uroradiologic imaging and to reduce invasiveness and radiation dose. As for the existing recommendations, the new proposals are consensus-based because evidence is lacking, use and indications have changed, or approaches on how to perform the examination in children differ in the literature (MRU). As in the previous recommendations, a thorough review of the literature and existing guidelines and recommendations has been performed. The proposals were discussed within the group and with nonmember experts and colleagues from other partner disciplines.
\end{abstract}

M. Riccabona $(\square)$

Department of Radiology, Division of Pediatric Radiology,

University Hospital Graz,

Auenbruggerplatz,

A-8036 Graz, Austria

e-mail: michael.riccabona@klinikum-graz.at

F. E. Avni

Department of Medical Imaging,

University Clinics of Brussels-Erasme Hospital,

Brussels, Belgium

J.-N. Dacher

Department of Radiology, CHU de Rouen,

Rouen, France

\section{B. Damasio}

Department of Radiology, Gaslini Institute,

Genoa, Italy
These recommendations aim to serve as a quality measure in order to standardise the procedures and thus grant comparable good quality results throughout different institutions.

Keywords Uroradiology - Recommendations · Procedures · IVU · MRU · Uro-CT · Child · Pediatric uroradiology ·

Children $\cdot$ Imaging

\section{Introduction}

The consistent work of the ESPR Paediatric Uroradiology taskforce and ESUR Paediatric Uroradiology working

K. Darge

Department of Radiology, Children's Hospital of Philadelphia,

Philadelphia, PA, USA

M. L. Lobo

Department of Radiology, Hospital de Santa Maria,

University Hospital,

Lisbon, Portugal

L.-S. Ording-Müller

Department of Radiology, University Hospital North Norway,

Tromsø, Norway

F. Papadopolou

Department of Radiology, Ioannina University Hospital,

Ioannina, Greece

U. Willi

Department of Radiology, John Hopkins University,

Baltimore, MD, USA 
group resulted in several published imaging and procedural recommendations over the last few years. These statements addressed important topics including imaging in prenatally diagnosed moderate and high-grade hydronephrosis $(\mathrm{HN})$, imaging in childhood urolithiasis and haematuria, imaging in childhood obstructive uropathy, imaging in childhood urinary tract infection (UTI) and procedural recommendations for urosonography and voiding cystourethrography (VCUG). Additionally, a statement has been issued on childhood aspects in nephrogenic systemic fibrosis (NSF) and a standardised adapted $\mathrm{HN}$ grading scheme has been presented. All these recommendations have since gained increasing acceptance throughout Europe and are also valued and applied in other continents.

To complete the existing procedural recommendations for common and important paediatric uroradiology procedures, the group has now addressed intravenous urography (IVU), uro-CT and MR-urography (MRU) in childhood and developed respective proposals. The aim of this process was again to standardise paediatric uroradiologic imaging, potentially reducing invasiveness and radiation, without taking risks and thereby missing conditions with potentially serious impact on individual morbidity. As for the existing recommendations, the new proposals are consensus-based because evidence is lacking (particularly on paediatric uro$\mathrm{CT}$ ), or use and indications have changed (IVU in childhood), or approaches on how to perform the examination in children differ in literature (MRU). As in the previous recommendations, a thorough review of the literature and existing guidelines and recommendations has been performed. The proposals were discussed within the group and with non-member experts and colleagues from other partner disciplines such as paediatricians, paediatric nephrologists, surgeons and urologists. Based on all the information obtained and the ensuing discussion within the group, the following three recommendations were drafted. We hope that they lead to standardised paediatric imaging applicable at least throughout Europe. This is a prerequisite to facilitate or allow comparable research results from different centres for meta-analysis and hopefully will reveal more evidence-based data for common paediatric uroradiological queries.

At the minisymposium session on "paediatric uroradiologic imaging and procedural recommendations, part III" at the annual ESPR meeting in Istanbul, Turkey in June 2009, these new proposals were presented and discussed, enabling a public opinion-making process. The results hereof and the feedback obtained at the guideline session of the annual ESUR meeting in Athens in September 2009, were included in the formulation of the final procedural recommendations.

In the following the final version of these recommendations is presented and concerns addressed that may still exist, reflecting unresolved issues from the discussions and future tasks in both standardisation as well as research. Scintigraphic techniques are not addressed, as they are well standardised and described by the paediatric nuclear medicine groups and committees.

\section{Intravenous urography in childhood}

IVU has been one of the mainstays in paediatric uroradiology for decades. Its use and typical findings have been well described and standardised. However, with established and widespread use of renal scintigraphy, the advent of MRU and the advances of US - particularly when applying modern methods and applications such as harmonic imaging, image compounding, high-resolution US or colour Doppler and contrast-enhanced sonography - the use of IVU decreased dramatically with few indications remaining. Thus, the procedural details of IVU need to be adapted accordingly.

This recommendation on performing IVU in children refers to circumstances in which IVU remains a diagnostic option, i.e. if MR is not available and/or US and VCUG yield insufficient information in view of the required treatment. While previously a large number of full abdominal exposures may have been part of routine IVU, the recommendation now is to reduce the radiation exposure to a minimum number and area in cases of need. This is usually accomplished by one to three properly timed and coned views (kidney, ureter, bladder film, KUB) based on the individual query and the results from all available previous imaging data, particularly from a mandatory detailed prior US study (see previous recommendation). The need for an initial abdominal plain film kidney-ureterbladder (KUB) radiograph should be decided according to the specific individual indication. Except for the question of urolithiasis, an initial KUB is rarely indicated. Persisting potential indications for an individualized childhood IVU are:

- Rare cases of pre- or postoperative imaging (with restricted access to MR)

- Suspected ureteral and renal trauma (if no CT available), or as a delayed KUB view after contrast-enhanced CT (avoiding a complementary delayed $\mathrm{CT}$ ) or in a child in intensive care

- Urolithiasis, if US is insufficient for therapy decision (see dedicated algorithm)

- Distinct pelvicalyceal or ureteral pathology (e.g., calyceal diverticula, early stages of medullary sponge kidney, ureteral valves)

The proposal includes a few technical aspects, addresses the above issues and insists on avoidance of unnecessary exposures or tomographic views (Fig. 1). Except for a table 
Fig. 1 IVU in childhood. NPO nil by mouth, $B W$ body weight, $C M$ contrast media, i.v. intravenous, $I V U$ intravenous urography, $K U B$ kidney-ureterbladder radiograph, $M R U \mathrm{MR}$ urography, US ultrasound
Prep
Pro
$\downarrow$

\section{Preparation}

age: minimum 4-6 weeks

infants: before next meal

NPO, hydration, creatinine, venous line ...

Procedure

adapt exposure \& contrast agent

proper use of filters, grid, shutters

renal area, or KUB (include symphisis)

initial KUB only if indispensable (e.g., urolithiasis)

reduced number exposures:

one early renal view at $5 \mathrm{~min}$

single late KUB at 15-20 min

additional focused images, if needed for treatment

diuretic IVU: Furosemide iv. $1 \mathrm{mg} / \mathrm{kg} \mathrm{BW}$ (max. $20 \mathrm{mg}$ )20 min before view

inject $5 \mathrm{~min}$ before, with, or 5-10 $\mathrm{min}$ after CM application

Goal: answer specific query with minimal radiation (ALARA)

$\Rightarrow$ avoid multiple, unnecessary or particularly tomographic views

with age- and weight-adapted volume of contrast material, no technical details are listed, since they are well established and known, i.e. proper filtering, inclusion of the pelvic floor in one film, or contrast-induced nephropathy (CIN) prevention rules. The given scheme was generally accepted, with some discussion on indications in pre-operative imaging, urolithiasis or trauma and to what extent the area of exposure might be restricted, e.g., in unilateral kidney or disease involvement. It was concluded that, apart from standard recommendations, individual tailoring of the examination to a given child's needs and symptoms as well as following individual institutional preferences and availability of equipment may be necessary (Figs. 2 and 3).

\section{CT of the urinary tract in children (paediatric uro-CT)}

CT has become the mainstay of uroradiology in adults. Particularly with modern multi-detector spiral CT and multiphase imaging its use increased for many applications beyond trauma, to infection, complicated cysts, masses and even routine investigation. The introduction of "low-dose" scanning techniques has boosted its use in urolithiasis, claiming that with proper settings the radiation dose be similar or even less than in IVU with a much higher yield of information. Recommendations on standardisation have been published. Increasingly, uro-CT is also used in the investigation of children. However, CT applies a relatively high radiation (particularly with multiphase acquisition) a particular consideration in children as they are more susceptible to radiation. In addition, diseases, tissue composition as well as size relationships differ in children.
According to the ALARA principle, a very different value and indication list results for paediatric uro-CT. Children are not small adults! As a rule, one should try to avoid CT in children whenever possible. Particularly if high-end US used by experienced operators and MRU is optimally exploited, most queries that may need CT-urography (CTU) in adults can be answered in children elsewhere, thus avoiding unnecessary radiation.

There are, however, childhood conditions that justify or require uro-CT. Therefore a proposal for performing uro$\mathrm{CT}$ in children was considered valuable in order to standardise imaging and to grant the lowest possible radiation burden. The recommendation on how to adapt and optimize a necessary CT of the paediatric urinary tract remains general. Because of the numerous different queries, the differences in equipment (e.g., number of detector rows, rotation time, detector sensitivity, tube specification, bore size) and the different clinical needs and approaches no single and universally applicable detailed protocol recommendation can be issued. Furthermore, there are only few hard data on the various applications in children and most of them describe the imaging findings in the various conditions rather than address the specific paediatric imaging technique and its restrictions, benefits and alternatives. Thus the proposal aims at offering some useful advice on the optimization of paediatric uro-CT examinations by adjusting the protocol considering lower ageadapted $\mathrm{kVp}$ and $\mathrm{mAs}$ settings, avoiding multiphase acquisitions, and choosing appropriate contrast amount and delay timing. The few accepted indications for uro$\mathrm{CT}$ in children are severe urinary tract trauma, complicated/ equivocal urolithiasis (if high level US+KUB are not conclusive and if there is expected therapeutic impact), 


\section{Indications}

- severe urinary tract trauma, complicated/equivocal urolithiasis \& infection, tumour \& DD, reno-vascular disease NOTE: only if high level US (+ KUB) not conclusive, always consider alternatively MRI, if available

\section{Preparation}

- avoid pain, decrease anxiety, local protection device, generous immobilisation \& sedation
- for CM administration -previous line placement, measure creatinine, hydration
NOTE: age dependent different normal creatinine values in infants \& children

\section{Contrast application}

- 2.5-1.0 ml/kg (weight dependent); injection speed 1-2ml/sec (if power injector applicable)

- age adapted injection rate \& scan delay time, hand or motor pump -depending on:

- location/size/type of IV access, child size/weight, underlying disease \& query

\section{Protocol}

NOTE: always use age-/weight-adapted paediatric settings, restrict acquisition area (scout \& scan!)

try to keep age corrected effective dose $<2 m S v$

tailor protocols to query (according to clinical indication \& result of previous US)

avoid multi-phase acquisitions

= plan \& perform CT-study according to query/clinical indication, including one ("or two/more") of the following: unenhanced, arterial, nephrographic, excretory phase

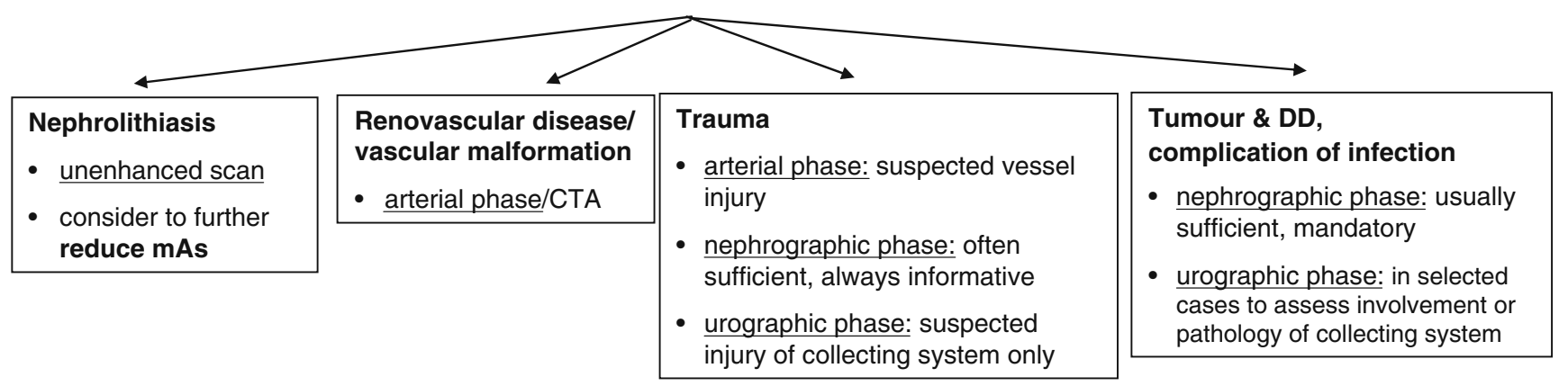

Fig. 2 Paediatric uro-CT. $D D$ differential diagnosis; $C M$ contrast media; $C T A C T$ angiography; $K U B$ kidney-ureter- bladder radiograph; $k V$ kilovolt; $m A s$ milliampere second; $m S v$ millisievert; $U S$ ultrasound

complicated infection (if US does not solve the query and MR is not available), tumour and its differential diagnosis (if MR is not available), and reno-vascular disease (if no interventional option is planned; otherwise, in patients with positive findings on Doppler sonography, consider proceeding directly to diagnostic catheter angiography with intervention in one session) (Fig. 2).

\section{Anatomic MR-urography in childhood}

MRU is a newer attractive imaging modality that was introduced to paediatric uroradiology more than a decade ago. It is by now an established non-ionising imaging modality for many queries in the child's urinary tract, and increasingly promoted as the ideal "one-stop shop" imaging for anatomic and functional queries. However, its availability is restricted, and many centres hesitate to apply the method to children, partially due to potential sedation needs, partially because of uncertainty in how to perform the investigation. Furthermore, discussions about NSF have hampered and prevented efforts, and multiple different approaches for imaging and for functional analysis have been reported in the literature, thus leading to confusion and hesitation regarding paediatric MRU. Therefore the group wants to promote the use of MRU in given paediatric queries trying to provide a safe and reliable MR protocol for anatomic imaging that is generally accepted and considered reliable. Since there is insufficient evidence and agreement on functional imaging approaches - in part also due to difference of surgical views regarding timing of the intervention, patient selection and differences in MR devices available - the group decided to address only the anatomic (semi-functional) imaging. A standardisation of functional diuretic MRU for quantitative renal functional evaluation and urinary drainage assessment will only be achievable once new studies and thorough comparison of the different approaches including discussion of their pros and cons in the different settings and age groups will be accomplished, and after extensive interdisciplinary consultations.

This proposal tries to include data from basic evaluation requirements such as preceding US information and, possibly, VCUG results. It addresses patient preparation and practical tips (e.g., when to catheterise, how to place 


\section{Indication}

Always previous US (+ reflux study, if indicated = VCUG, ce-VUS, or RNC)

Queries: e.g. malformation, obstructive uropathy, complicated infection, tumour, post-traumatic, cystic disease, transplant ...

\section{Preparation}

- General: Place line in advance, creatinine for CM-studies (GFR calculation -NSF), mock unit / visit to magnet

- Hydration: NaClor Ringer's solution (20 ml/kg for 1 hour [maximum 11]), empty bladder before entering the magnet

- Sedation: priority to immobilization (feed \& wrap), or no (minimal) sedation. Deep sedation only if necessary

- Bladder catheter: deeply sedated patients who cannot empty the bladder (particularly after Furosemide)

- potentially also in high grade VUR with dynamic queries

- Polyethlene catheter without balloon, urine bag, below level of MR table

- Diuresis: Furosemide $1 \mathrm{mg} / \mathrm{kg}$ iv. (max. $20 \mathrm{mg}$ ), $15 \mathrm{~min}$ before to beginning of morphologic investigation

- timing may vary in dynamic-diuretic functional protocols $(F-20, F-15, F 0, F+10, F+15, F+20)$

\section{MRU examination*1}

Positioning Supine position with arms above the head

Scout Sagittal important for correct oblique coronal plane, FOV: from above both diaphragms to below symphysis

- potentially SSFP axial \& coronal (+ sagittal)

Heavily T2-weighted sequences coronal (e.g.,T2-3D TSE fs or 2D-thin \& -thick slice [3D-UROGRAM], HASTE/RARE/PACE, ...)?

T2-IR sequence, non-enhanced T1-weighted \& GRE sequence

- NOTE: 3 slices anterior + posterior of kidneys for GRE; adjust FOV

CM-Application - cyclic Gd compounds ${ }^{2}$ iv. in first year of life (renal immaturity...) \& bilateral uropathy, or GFR $\downarrow$

Repeated serial coronal T1-3D GRE fs (for functional assessment continuously for 35min.)

- NOTE: subtraction helpful - particularly for MRA, if achievable; for MRA use motor pump \& flow of $1 \mathrm{ml} / \mathrm{sec}$

T1 axial \& coronal (fs), + sagittal if needed

Final coronal T1-3D GRE fs; or additional delayed imaging up to 20(-30) min p.i.

- potentially changing to prone position or post void scan (when delay in CM washout)

\footnotetext{
*1 functional MRU not yet standardised and not addressed

- Furosemide timing, contrast dose \& application may need adaptation

for various queries tailored protocols are essential

- e.g., MRA, diffusion, additional sagittal acquisition
}

Fig. 3 Anatomic paediatric MRU. ce-VUS contrast-enhanced voiding urosonography; $C M$ contrast media; $D D$ differential diagnosis; $F$ furosemide; FOV, field of view; $f s$ fat saturation; $G F R$, glomerular filtration rate; $G R E$ gradient-echo (sequence); $G d$ gadolinium; $I R$ inversion recovery (sequence); iv intravenous; $m g$ milligram; min

the urine bag, sedation and immobilisation needs, hydration and diuretic stimulation) as well as NSF precautions. The typical MRU protocol based on the non-enhanced, heavily T2-weighted "water-MRU" sequences is detailed, including suggestions in how to plan and orient the acquisition, before finally contrast-enhanced serial T1-weighted gradient-echo sequences as well as other standard T1-W imaging before and after gadolinium administration are acquired to complete the investigation - these will allow for visual assessment of contrast uptake and elimination, renal function and contrast excretion as well as drainage from the collecting system (Fig. 3).

\section{Conclusion}

This proposal completes the procedural recommendations in paediatric uroradiology. They aim to serve as a quality measure in order to standardise the procedures and thus grant comparable good quality results throughout different
${ }^{* 2}$ non-cyclic compounds can be used in older children according to approval - Gd-dose as recommended by manufacturer

minutes; $M R A$ MR-angiography; $\mathrm{NaCl}$ physiologic saline; $N S F$ nephrogenic systemic fibrosis; p.i. after injection; $R N C$ radionuclide cystography; SSFP steady state free precession (sequence); TSE turbo spin-echo (sequence); US ultrasound; $V C U G$ voiding cystourethrography; VUR vesico-ureteral reflux

institutions. It is also hoped that they will, in the future, allow the creation of more evidence for deciding on impact and necessity of the various imaging options in the various childhood conditions of the kidneys and urinary tract. Of course, with new knowledge, evidence and insight and new advances in imaging techniques and potential, recommendations and guidelines will have to be adapted, just as procedural techniques might need adaptation.

\section{References}

1. Akay H, Akpinar E, Ergun O et al (2006) Unenhanced multidetector. CT evaluation of urinary stones and secondary signs in pediatric patients. Diagn Interv Radiol 12:147-150

2. Dacher JN (2008) Diagnostic procedures excluding MRI, nuclear medicine and video urodynamics. In: Fotter $\mathrm{R}$ (ed) Pediatric uroradiology, 2nd edn. Springer, Berlin, p 10

3. Dyer RB, Chen MY, Zagoria RJ (2001) Intravenous urography: technique and interpretation. RadioGraphics 21:799-821

4. Grattan-Smith JD, Little SB, Joones RA (2008) MR urography in children: How we do it. Pediatr Radiol 38:S3-S17 
5. Karmazyn B, Frush DP, Applegate KE et al (2009) CT with a computer-simulated dose reduction technique for detection of pediatric nephroureterolithiasis: comparison of standard and reduced radiation doses. AJR 192:143-149

6. Riccabona M, Avni FE, Blickman JG et al (2008) Imaging recommendations in paediatric uroradiology: minutes of the ESPR workgroup session on urinary tract infection, fetal hydronephrosis, urinary tract ultrasonography and voiding cysto-urethrography. Barcelona, Spain, June 2007. Pediatr Radiol 38:138-145

7. Riccabona M, Avni FE, Blickman JG et al (2009) (Members of the ESUR paediatric paediatric recommendation work group and ESPR paediatric uroradiology work group). Minutes of the ESPR uroradiology task force session on childhood obstructuve uropathy, high-grade fetal hydronephrosis, childhood haematuria, and urolithiasis in childhood. ESPR Annual Congress, Edinburgh, UK, June 2008 Pediatr Radiol 39:891-898
8. Rohrschneider WK, Haufe S, Wiesel M et al (2002) Functional and morphologic evaluation of congenital urinary tract dilatation by using combined static-dynamic MR urography: findings in kidneys with a single collecting system. Radiology 224:683-694

9. Shine S (2008) Urinary calculus: IVU vs. CT renal stone? A critically appraised topic. Abdom Imaging 33:41-43

10. Smergel E, Greenberg BS, Crisci KL et al (2001) CT urograms in pediatric patients with ureteral calculi: do adult criteria work? Pediatr Radiol 31:720-723

11. Strouse PJ, Bates DG, Bloom DA et al (2002) Non-contrast thinsection helical CT of urinary tract calculi in children. Pediatr Radiol 32:326-332

12. Van Der Molen AJ, Cowan NC, Mueller-Lisse UG et al (2008) (CT Urography Working Group of the European Society of Urogenital Radiology). CT urography: definition, indications and techniques. A guideline for clinical practice. Eur Radiol 18:4-17 